\title{
ERRATUM \\ LIKELIHOOD RATIO AND HAZARD RATE ORDERINGS OF THE MAXIMA IN TWO MULTIPLE-OUTLIER GEOMETRIC SAMPLES-ERRATUM
}

\author{
BaOJUN Du \\ School of Mathematics and Statistics \\ Lanzhou University, Lanzhou 730000, China \\ Peng Zhao \\ School of Mathematical Sciences \\ Jiangsu Normal University, Xuzhou 221116, China \\ N. BALAKRISHNAN \\ Department of Mathematics and Statistics \\ McMaster University, Hamilton, Ontario, Canada L8S 4K1 \\ E-mail:bala@mcmaster.ca
}

(Probability in the Engineering and Informational Sciences, 26, 2012, 375-391. doi:10.1017/S026996481200006X)

The e-mail address for Dr. Peng Zhao was removed from the article in error. Below is the correct contact information for Dr. Zhao.

\section{Peng Zhao}

School of Mathematical Sciences

Jiangsu Normal University, Xuzhou 221116, China

E-mail: zhaop07@gmail.com 